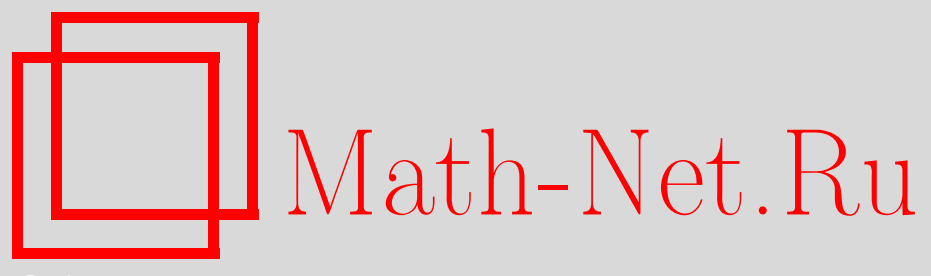

В. М. Бабич, А. Р. Итс, В. А. Марченко, Л. А. Пастур, Б. А. Пламеневский, Т. А. Суслина, Л. Д. Фаддеев, А. А. Федотов, Н. Н. Уральцева, Владимир Савельевич Буслаев (некролог), УМH, 2014, том 69, выпуск 1, 163168

DOI: https://doi.org/10.4213/rm9558

Использование Общероссийского математического портала Math-Net.Ru подразумевает, что вы прочитали и согласны с пользовательским соглашением http://www . mathnet.ru/rus/agreement

Параметры загрузки:

IP : 54.198 .187 .58

26 апреля 2023 г., 13:08:28

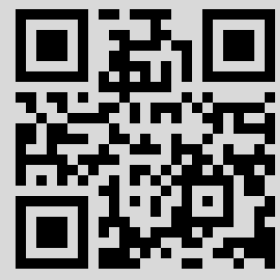




\section{Владимир Савельевич Буслаев}

Владимир Савельевич Буслаев ушел из жизни 14 марта 2012 г., за месяц до своего семидесятипятилетия. Он был ярким ученым, одним из лидеров Ленинградской-Петербургской математической школы и заслужил широкое международное признание.

Владимир Савельевич был удивительно разносторонним и глубоким математиком: он получил принципиальные результаты в целом ряде областей, среди которых - формулы следа в квантовых и классических задачах, дифракция и распространение волн, квантовая теория рассеяния, асимптотический анализ, вполне интегрируемые нелинейные уравнения, квазиклассические псевдодифференциальные операторы с разрывными символами, разностные уравнения с периодическими коэффициентами и теория устойчивости солитонных решений для неинтегрируемых

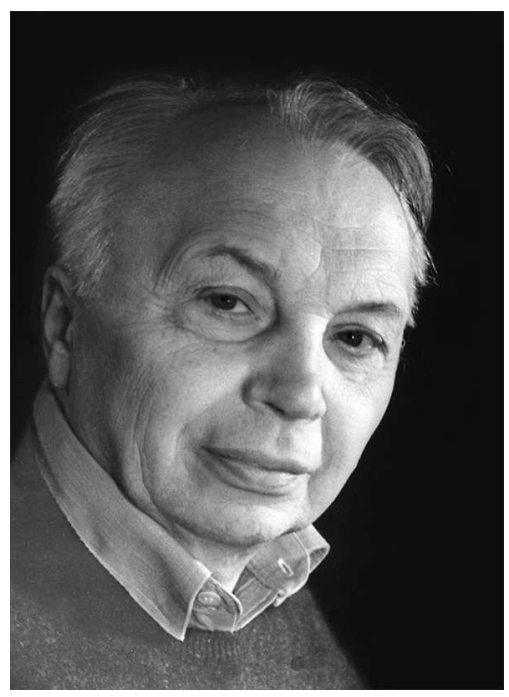
нелинейных уравнений. При этом ему принадлежит приоритет в открытии и разработке ряда научных направлений, а его работы послужили отправной точкой для нескольких направлений исследований.

В 1959 г. Владимир Савельевич окончил физический факультет ЛГУ, в 1959-1962 гг. проходил обучение в аспирантуре ЛГУ. Учителями В. С. Буслаева были Ольга Александровна Ладыженская и Людвиг Дмитриевич Фаддеев. В 1963 г. он защитил кандидатскую диссертацию "Коротковолновая асимптотика в задаче дифракции на выпуклых телах", а в 1973 г. - докторскую диссертацию "Спектральные асимптотики и формулы следа для уравнения Шрёдингера".

В течение 50 лет, с 1962 г. и до конца своих дней, Владимир Савельевич работал на кафедре высшей математики и математической физики ЛГУ (после перестройки СПбГУ), а последние 12 лет возглавлял ее.

Владимир Савельевич был блестящим педагогом высочайшей математической культуры. Он - автор замечательного учебника по вариационному исчислению.

Владимир Савельевич всегда искал оригинальные аналитически богатые задачи, пути и результаты исследования которых было трудно предсказать заранее. Как старые мастера, он любил ручную работу с формулами, путь к общим конструкциям теории для него лежал через анализ сложных конкретных задач. Такой подход он считал одной из главных отличительных особенностей Петербургской математической школы, и сам обладал редким талантом "аналитика-формулиста". Он живо

DOI: $10.4213 / \mathrm{rm} 9558$ 
реагировал на чужие идеи и результаты, старался все понять по существу и взглянуть на работы "сверху", поделиться своим видением с коллегами. Им был создан уникальный научный семинар, посвященный проблемам спектрального и асимптотического анализа, и на протяжении десятков лет он руководил его работой. Владимир Савельевич воспитал целую плеяду учеников, многие из которых сами стали известными учеными. Своим ученикам В. С. Буслаев старался предлагать открывающие перспективы задачи. Обладая редкой математической интуицией, Владимир Савельевич выбирал проблемы, с которыми не могли справиться известные специалисты, и давал их своим питомцам, говоря, что это - "задачи нашей аналитической культуры", и задачи решались.

В.С. Буслаева постоянно приглашали в ведущие университеты, международные научные центры и на международные конференции. В 1975 г. за цикл работ по теории дифракции Владимир Савельевич Буслаев был удостоен первой премии Ленинградского государственного университета за лучшие научные исследования. Заслуги В. С. Буслаева отмечены Государственной премией РФ в области науки и техники (2000), почетными званиями "Заслуженный работник высшей школы РФ" (1999), "Заслуженный деятель науки РФ" (2008), званием "Почетный профессор СПбГУ" (2012). В 2005 г. Университетом Paris Nord ему была присвоена почетная степень Doctor Honoris Causa. В. С. Буслаев был членом редколлегий журналов РАН "Алгебра и анализ" и "Функциональный анализ и его приложения".

В. С. Буслаев - автор более чем 150 работ. Коротко опишем его основные научные достижения.

Формулами следа называют тождества, связывающие спектральные характеристики дифференциального оператора с функционалами от его коэффициентов. В 1960 г. были опубликованы ставшие знаменитыми формулы следа Буслаева-Фаддеева. Авторами был, по-видимому впервые, строго получен полный набор формул следа для одномерного оператора Шрёдингера с непрерывным спектром (оператор на полуоси). Позже Владимир Савельевич распространил результаты этой работы на многомерный случай, что потребовало развития очень продвинутого аналитического аппарата. В начале 1970-х годов Владимир Савельевич вновь вернулся к формулам следа уже в связи с квантовой трехчастичной задачей. Это было непростым делом: формулы, опубликованные до него, оказались некорректными. В начале $1970-$ х годов им были получены формулы следа для модели Фридрихса, затем для оператора, который можно рассматривать как "трехчастичный" аналог модели Фридрихса, и, наконец, обобщение развитых методов позволило ему успешно исследовать систему трех одномерных частиц. Одновременно вместе со своим учеником С. П. Меркурьевым он получил формулы следа для трех трехмерных частиц. При этом содержательно использовались результаты Л. Д. Фаддеева о трех частицах (1963) и работы М. Ш. Бирмана и М. З. Соломяка об оценках сингулярных чисел интегральных операторов (1967).

Владимиру Савельевичу принадлежит замечательное по своей естественности оправдание геометро-оптического приближения в задаче рассеяния волн на компактном гладком выпуклом теле в освещенной области и полутени. Его внимание к этим исследованиям привлекла Ольга Александровна Ладыженская. Стандартный подход работал лишь в случае условия Дирихле. Сложнейшая задача, которую начал решать еще Ф. Эрселл (1957), была решена в работах В. С. Буслаева к середине 1970-х годов. Отметим, что к ним вплотную примыкает серия статей В. С. Буслаева о формулах следа и об асимптотиках спектральных характеристик при больших значениях спектрального параметра для эллиптических операторов во внешних областях. Говоря о дифракции на гладком выпуклом теле, упомянем и изящную работу В. С. Буслаева об эвристическом выводе (очень непростых!) асимптотик волнового поля для этой задачи с помощью интеграла Винера. 
В середине 1960-х годов Владимир Савельевич предложил производящий интеграл - обобщение метода Вентцеля-Крамерса-Бриллюэна, эквивалентное каноническому оператору Маслова. При его построении вместо формальных асимптотических рядов метода Вентцеля-Крамерса-Бриллюэна, для которых выделенную роль играет координатная плоскость в фазовом пространстве классической системы, В. С. Буслаев рассматривал их обобщения, связанные с лагранжевыми плоскостями, касающимися лагранжева многообразия.

Широкую известность получили работы В. С. Буслаева по рассеянию на дальнодействующих потенциалах и его глубокие исследования по аналитической теории многочастичного рассеяния. Для операторов Шрёдингера с медленно убывающими потенциалами пределы, определяющие волновые операторы в традиционной теории рассеяния, не существуют. В случае кулоновского потенциала аналог волнового оператора построил J.D. Dollard. В.С. Буслаев и Л. Д. Фаддеев сформулировали гипотезу об общем виде волновых операторов. В. С. Буслаев и В. Б. Матвеев завершили конструкцию обобщенных волновых операторов и доказали их существование. В 1974 г. вместе с М. М. Скригановым Владимир Савельевич построил полные формальные асимптотические разложения решения задачи рассеяния для трехмерного уравнения Шрёдингера на бесконечности для некоторого класса убывающих потенциалов. Полученные формулы имели естественную дифракционную интерпретацию, а сама работа предваряла развитый позже дифракционный подход к задаче рассеяния нескольких квантовых частиц. В 1979 г. с его помощью Владимир Савельевич с С.П. Меркурьевым и С.П. Саликовым исследовал рассеяние в системе трех одномерных квантовых частиц с быстро убывающими четными парными потенциалами. Позже вместе с Н. А. Калитеевским он изучил случай $n$ одномерных квантовых частиц. В конце 2000-х годов Владимир Савельевич вместе с С. Б. Левиным обратился к рассеянию трех кулоновских трехмерных квантовых частиц. Случай быстро убывающих парных потенциалов был изучен Л. Д. Фаддеевым в 1963 г. Для исследования медленно убывающих парных потенциалов В. С. Буслаев и С. Б. Левин использовали идею дифракционного подхода и получили очень непростые формальные асимптотики решения задачи рассеяния.

Серия работ Владимира Савельевича посвящена формулам следа, которые связывают регуляризованные определители линейных дифференциальных операторов, порождаемых уравнениями в вариациях для классических механических систем, с якобианами преобразований, задаваемых движениями этих систем. Лагранжев вариант формулы следа был описан В. С. Буслаевым в 1968 г. В 1982 г. В. С. Буслаев и Е. А. Рыбакина получили формулу следа для гамильтоновой системы. Позже формулы следа были ими существенно обобщены, а в конце 1980-х годов авторы вывели формулы следа для системы с бесконечным числом степеней свободы - для скалярного релятивистского поля, описываемого нелинейным уравнением Клейна-Гордона.

Вычисление временных асимптотик решений задач Коши для интегрируемых уравнений является одним из наиболее впечатляющих достижений метода обратной задачи в теории солитонов. Соответствующий аппарат, начиная с пионерских работ В.Е. Захарова и С. В. Манакова и завершая работами П. Дейфта и Ж. Жоу по нелинейному методу перевала, разрабатывался усилиями многих исследователей в течение почти двадцати лет. Работы Владимира Савельевича занимают специальное место в истории создания современной асимптотической теории интегрируемых систем. В них впервые была строго выведена временная асимптотика и выявлены ее теоретико-операторное значение и гамильтонов смысл. В. С. Буслаев вместе с В. В. Сухановым занимался уравнением КдФ. В 1982-1985 гг. они получили асимптотические формулы для решения задачи Коши с быстро убывающими начальными данными, существенно уточнившие предшествующие эвристические результаты. 
В частности, была впервые получена точная формула для асимптотической фазы решения. Тогда же были введены волновые операторы. Вскоре после этого В. С. Буслаев, Л. А. Тахтаджян и Л. Д. Фаддеев использовали описанные асимптотики для построения переменных типа действие-угол. Это привело к элегантной гамильтоновой интерпретации теории рассеяния.

В 1980-е годы Владимир Савельевич занимался задачами акустики океана. Распространение звука в реальном океаническом волноводе описывается уравнением Гельмгольца с большим параметром, ведущим к коротковолновым асимптотикам. При этом поле асимптотически формируется как сумма вкладов лучей. Владимир Савельевич заметил, что если источник и приемник звука находятся вблизи поверхности глубокого океана, то все соединяющие их лучи естественно разбиваются на четверки с близкими набегами фазы, а вклады четверок описываются простыми асимптотическими формулами. "Четверочные формулы” позволили детально объяснить наблюдаемую экспериментаторами сложную интерференционную структуру волнового поля. Другая известная серия результатов касается рассеяния коротких волн на адиабатической неоднородности в плоском слоистом волноводе с границами. Эта модель описывает рассеяние звука на синоптических вихрях в океаническом волноводе. Задача привлекала внимание известных специалистов. В.С. Буслаев и А. А. Федотов асимптотически вычислили матрицу прохождения нормальных волн через неоднородность и показали, что ее асимптотическая структура связана с точностью сохранения адиабатического инварианта вдоль лучей, рассматриваемых как траектории гамильтоновой системы, медленно зависящей от времени. Выяснилось, что наличие у волновода границ приводит к появлению лучей, вдоль которых изменения адиабатического инварианта относительно велики, и, соответственно, к недиагональности матрицы прохождения уже в старших порядках.

В середине 1980-х годов Владимир Савельевич занялся изучением эффектов, вызванных адиабатическими возмущениями линейных дифференциальных уравнений с периодическими коэффициентами. Так, серия работ В. С. Буслаева и его совместных работ с Л.А. Дмитриевой и А. Грижисом была посвящена одномерному уравнению Шрёдингера $-d^{2} \psi / d x^{2}+(V(x)+W(\varepsilon x)) \psi=E \psi$, где $V$ - периодическая функция, a $\varepsilon$ - малый параметр. Интерес к таким задачам возникал, например, в физике твердого тела. В работах В.С. Буслаева был развит оригинальный мощный асимптотический подход, позволяющий эффективно их исследовать. Отметим, что после замены переменной $x \rightarrow \xi=\varepsilon x$ выписанное выше уравнение Шрёдингера с $V=0$ преобразуется к виду $-\varepsilon^{2} d^{2} \psi / d \xi^{2}+W(\xi) \psi=E \psi$, параметр $\varepsilon$ оказывается стандартным квазиклассическим параметром, и можно сказать, что обычные квазиклассические методы позволяют исследовать уравнения, возникающие при добавлении адиабатического возмущения $W(\varepsilon x)$ к оператору $-d^{2} / d x^{2}$. Метод Буслаева является глубоким обобщением: он позволяет исследовать адиабатические возмущения нетривиального периодического оператора $-d^{2} / d x^{2}+V(x)$. Развитые общие конструкции были с успехом применены для решения тонкой аналитической задачи, которой пытались заниматься многие специалисты, - исследования геометрии резонансов Штарка-Ваннье в адиабатическом приближении.

С середины 1980-х годов В. С. Буслаев и А. М. Будылин вместе занимались исследованиями квазиклассических псевдодифференциальных уравнений с разрывными символами. Первая задача пришла из теории информации. Авторами были найдены асимптотические разложения при $\varepsilon \rightarrow 0$ следов гладких функций оператора $A_{\varepsilon}$, определенного формулой $\left(A_{\varepsilon} f\right)(x)=\varepsilon^{-d} \int_{\Omega} A((x-y) / \varepsilon) f(y) d y$, где $\Omega$ - ограниченная область в $\mathbb{R}^{d}$ с гладкой границей. Для старших коэффициентов асимптотических разложений были получены явные формулы, существенно зависящие от геометрии 
области $\Omega$ и ее границы. Следующим шагом стал асимптотический анализ решений уравнений вида $\left(B_{\varepsilon} f\right)(x)=g(x)$, где $\left(B_{\varepsilon} f\right)(x)=\varepsilon^{-1} \int_{-1}^{1} B((x-y) / \varepsilon, x, y) f(y) d y$, в случае наличия скачков и/или нулей по всем переменным в символе $b(\xi, x, y)=$ $\int B(u, x, y) \exp (-i u \xi) d u$. В серии работ В. С. Буслаев и А. М. Будылин построили асимптотическую теорию таких квазиклассических псевдодифференциальных уравнений с приложениями к ряду задач математической физики. Развитые методы оказались применимы и к операторам более общего типа, формально принадлежащим к классу интегральных операторов Фурье, и в начале 2000-х годов авторами была найдена асимптотика фредгольмова детерминанта, определяющего двухчастичную корреляционную функцию для Бозе-газа одномерных частиц, а позже была начата разработка альтернативного к нелинейному методу перевала Дейфта и Жоу подхода к асимптотическому решению матричных задач сопряжения, возникающих при решении задач Коши для интегрируемых уравнений.

В 1991-2008 гг. Владимир Савельевич в соавторстве с Галиной Перельман, а затем с Катрин Сулем совершил важнейшие открытия в теории устойчивости солитонов (устойчивость односолитонных решений к малым возмущениям). Исследовалось одномерное нелинейное уравнение Шрёдингера с нелинейностью вида $F\left(|u|^{2}\right)$ вместо $|u|^{2}$, и был развит геометрически прозрачный подход, позволивший доказать асимптотическую устойчивость солитонов. До этого удавалось обнаружить лишь орбитальную устойчивость солитонов, а асимптотическая была установлена лишь для уравнения с дополнительным линейным слагаемым с коэффициентом, зависящим от переменной уравнения. Развитый подход оказался универсальным и с успехом применялся другими авторами для решения широкого круга задач.

В 1992-2000 гг. В. С. Буслаев вместе с А. А. Федотовым изучал разностные уравнения, рассматриваемые на вещественной оси или комплексной плоскости и имеющие периодические коэффициенты. Такие уравнения возникли в физике твердого тела и привлекли внимание своими богатыми и необычными спектральными свойствами. Авторами был открыт метод монодромизации - очень естественный перенормировочный подход к исследованию периодических разностных уравнений. Он возник на пути переосмысления основных объектов теории дифференциальных уравнений с периодическими коэффициентами. Оказалось, что попытка построить блоховские решения для разностных уравнений приводит к бесконечной цепочке разностных уравнений, подобных исходному. Возникает динамическая система, осуществляющая "пересчет" коэффициентов каждого из уравнений цепочки в коэффициенты следующего за ним. Можно сказать, что появление такой динамической системы отражает канторовость спектра обсуждаемых уравнений. Анализ свойств исходного уравнения за счет анализа свойств этой системы и есть ведущая идея метода монодромизации. В работах авторов было создано новое аналитическое направление исследования разностных уравнений. Позже идеи метода монодромизации стали важным инструментом исследования разностных и дифференциальных квазипериодических уравнений.

В последнее свое десятилетие, работая над задачей рассеяния трех заряженных квантовых частиц и продолжая исследования по теории псевдодифференциальных операторов с разрывными символами, Владимир Савельевич начал серию новых проектов. Коротко обсудим несколько из них. Вместе Л. А. Пастуром В. С. Буслаев исследовал ансамбли больших случайных эрмитовых матриц, связанные с моделями из квантовой теории поля. Он начал исследование интригующей задачи - оператора Штарка-Ваннье с периодическим потенциалом Кронига-Пенни (периодическая цепочка $\delta$-функций) - и нашел ее нетривиальную и глубокую редукцию. Вместе с Е. А. Грининой и с К. Сулем он опубликовал несколько статей, посвященных исследованиям адиабатической эволюции, порожденной линейными операторами, завися- 
щими от времени. Под влиянием работ М.Ш. Бирмана и Т. А. Суслиной Владимир Савельевич обратился к задачам теории гомогенизации и вместе со своим учеником А. А. Пожарским исследовал рассеяние волн на периодических структурах.

Владимир Савельевич активно работал буквально до последнего дня: умер он по дороге на научный семинар.

В. М. Бабич, А.Р. Итс, В.А. Марченко, Л. А. Пастур, Б.А. Пламеневский, Т.А. Суслина, Л.Д. Фаддеев, А.А. Федотов, Н.Н. Уральцева 